\title{
Assessment of the Bio Preservative Efficacy of Trametes polyzona (pers.) Extracts on Tomato Fruits
}

\author{
Oduntan, O.O \\ ${ }^{1}$ Fruits Programme, National Horticultural Research Institute, \\ P.M.B. 5432, Idi-Ishin, Ibadan, Oyo State, Nigeria.
}

\begin{abstract}
The present study examined the biopreservative efficacy of Trametes polyzona extracts on tomato fruits. Microorganisms associated with the spoilage of tomato fruits were isolated and identified using standard microbiological methods. Pathogenicity test of all the microbial isolates were also done using standard techniques. Qualitative and quantitative phytochemical constituents of Trametes polyzona was also assessed. Moreover, antimicrobial effect and preservative potential of the extracts of Trametes polyzona on microbial isolates of tomato and the tomato fruits were assessed respectively. The proximate composition of the fresh and preserved tomato fruits were further evaluated and compared after storage. Result obtained from the microbial analysis revealed that Staphylococcus aureus, Bacillus spp, Escherichia coli, Klebsiella spp, Lactobacillus spp, Shigella spp, Pseudomonas spp, Micrococcus spp, Rhizopus stolonifera, Aspergillus niger, A.terreus, A. flavus, and Fusarium spp were found in the spoilt tomato fruits. Qualitative phytochemical screening showed saponin, tannin, phytate, flavonoid, terpenoid, total phenol, and oxalate available in Trametes polyzona extracts, while anthraquinone and phlobatannin were absent. There were variations in the quantity of the phytochemicals present in each extract with values ranging from $(0.03$ to $9.61 \mathrm{mg} / \mathrm{g}$ ). $\mathrm{N}$-hexane extract of showed inhibitory effect on all the bacterial isolates with zones of inhibition ranging from $(2.04$ to $27.00 \mathrm{~mm})$. The extracts were not able to inhibit the fungal isolates. At the sixth week of storage, tomato fruits preserved with $n$-hexane extract and kept in the refrigerator were observed to retain their firmness and texture. Moisture content of preserved tomato fruits were lower than fresh tomato fruits, while ash, fibre, protein and carbohydrate content of the preserved tomato fruits was observed to be higher than fresh tomato fruits. Vitamin $\mathrm{C}$ content of the preserved tomato fruits $(66.41 \mathrm{mg} / \mathrm{g})$ was also higher than the fresh tomato fruits $(38.58 \mathrm{mg} / \mathrm{g})$. In conclusion, N-hexane extracts of Trametes polyzona demonstrated the highest inhibitory potential against all the spoilage bacteria associated with tomato fruits, together with a significant preservative potential on tomato fruits compared to other extracts of Trametes polyzona. The results from this study revealed that $\mathrm{N}$-hexane may be used to extend the shelf life of tomato fruits and keeping at refrigeration temperature can further help in preserving tomato.
\end{abstract}

Keywords:- Bio-Preservative, Phytochemical Screening, Trametes polyzona Extracts, Tomato Fruits, Shelf Life.

\section{INTRODUCTION}

Tomatoes may be pear-shaped, elongated, flattened and heart shaped. They are edible, fleshy and reddish when ripe and vary in their acid composition, with white and yellow ones being less acidic. Tomatoes can be used as savoury or flavouring in soups and cooked foods or can be eaten as fruits. It is used in many dishes, salads, sauces and drinks and can also be dried and ground into pancakes (Effiuvwevwere, 2000). According to the Food and Agriculture Organization (FAO, 2013), global tomato production (processing and fresh) reached 162 million metric tons in 2012, while global trade increased to \$59 billion. Nigeria is ranked $16^{\text {th }}$ in the world with the estimated production capacity of 1.8 million metric tons and second highest producer in Africa behind Egypt (FAO, 2013).

Tomato has a much lower sugar content than other fruits and is therefore not as sweet, it is rich in vitamins including vitamin $\mathrm{A}$ and vitamin $\mathrm{C}$, carbohydrates, proteins, fats, fibres, potassium, phosphorus, iron and lycopene which has many beneficial health effects (Naika et al., 2005, Talvas et al., 2010). The consumption of tomatoes throughout the world is believed to benefit the heart and other organs. Tomatoes are the primary source of lycopene in many people's diets (Slimestad and Verheul, 2005). Lycopene is also responsible for the red colour present in the tomato (Sabio et al., 2003). Lycopene has been found to prevent prostate cancer, improve the skin's ability to protect itself against the harmful ultra violet rays, decrease the risk of breast, lung, stomach, bladder, uterine, head and neck cancers, protect against neurodegenerative diseases, lower urinary tract infections and reduce thecardiovascular risk associated with type 2 diabetes (Freedman et al., 2008; Zhang et al., 2009; Shidfar et al., 2010; Zdenka et al., 2010). It contains large amount of water which makes it more susceptible to spoilage by the action of microorganisms particularly fungi and bacteria (Bai and Lindhout; 2006, Samuel and Orji, 2015). 
ISSN No:-2456-2165

Spoilage of tomatoes are those adverse changes in the quality of tomatoes that are brought about by the action of predominantly biological and physical factors. These may be changes in taste, smell, appearance or texture of the fruits. Ghosh (2009) reported that fungi were the source of spoilage of most of the tomato samples assessed than bacteria. Fungi affecting tomatoes include; Trichoderma spp, Alternariaalternata, Fusariumoxysporum, Fusariummoniliformis, Aspergilliusniger, Mucor spp, Rhizopusstolonifer, Penicillium spp, Geotrichum spp and Phytophthora spp (Fatih et al., 2005; Akinmusire, 2011; Akintobi et al., 2011).

The macrofungus Trametes spp (plate 1) is polyporoid white rot fungi widely distributed in various biotopes and have been the subject of many physiological and biochemical studies (Koroleva et al., 2002).

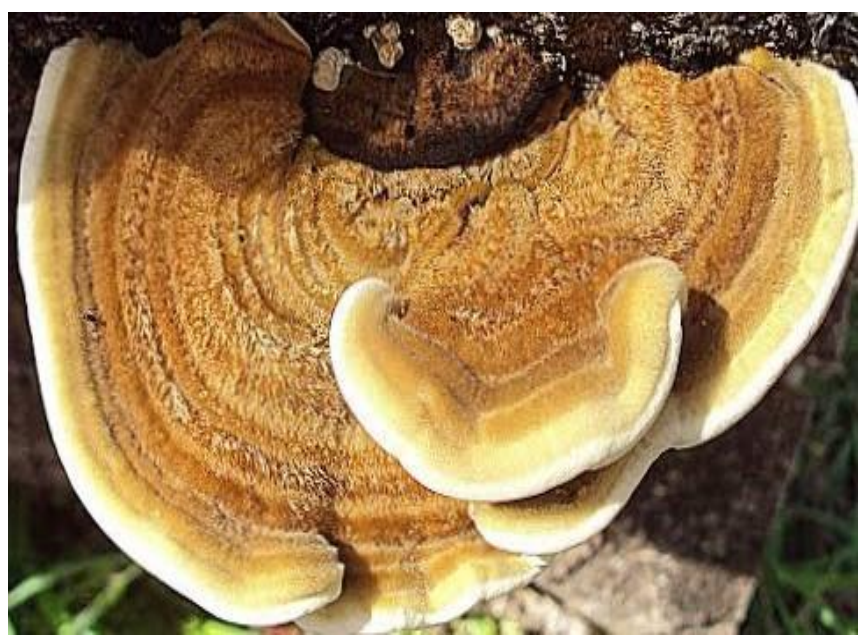

Plate 1:- Photography of Trametes polyzona

One of the limiting factors that influence tomato economical value is its relatively short shelf life caused by pathogen attack, inadequate handling and poor preservation methods (Samuel and Orji, 2015). In recent decades, use of synthetic preservatives in food preservation and processing has been a common practice to control foodborne microorganisms. However, a public antipathy has developed recently against synthetic chemicals in foods due to consumer anxiety over pesticides residues on food, and also the ecological damage caused by the chemical (Nicholson, 2007). Therefore, discovering and applying natural antimicrobials especially from traditional food materials such as edible mushrooms (macrofungi) has become attractive to researchers and the food industry (Shen et al., 2017). Mushrooms are known to contain useful bioactives with potential preservative properties (Oyetayo et al., 2010). Although the reports on successful applications of mushroom antimicrobials as food safety control agents are considerably few, and more studies on the use of mushroom antimicrobials for food safety purposes has been increasing significantly (Gao et al., 2005; Alves et al., 2012). Hence, this present study aim at examining bio-preservative efficacy of Trametes polyzona extracts on tomato fruits.

\section{MATERIALS AND METHODS}

\section{$>$ Collection of tomato fruits}

The study was carried out in Ondo city and Akure metropolis of Ondo state. Tomato fruits were collected from retailers in three different market in Akure namely; Ojaoba market, Isikan market, and FUTA north gate. The samples from each location were collected in a sterile plastic container identified with codes and transferred to Microbiology Postgraduate Laboratory, Federal University of Technology, Akure for analyses.

\section{Collection of mushroom}

The fruiting bodies of mushroom, Trametes polyzona was collected from the FUTA forest, while identity of the mushroom was authenticated at the Department of Microbiology, Federal university of Technology, Akure, (FUTA) Ondo State.

\section{$>$ Culture media}

The culture media used for this research work are Nutrient agar, Nutrient broth, Eosin methylene blue (EMB) agar, MacConkey agar, Salmonella-Shigella agar, Potato dextrose agar and Mueller Hinton agar. Each of the culture medium was prepared and sterilized according to the manufacturer's specification.

\section{Glass ware}

The glassware used in this research includes petri dishes, test tubes, conical flasks, beakers and Mac Cartney bottles. They were washed, left to dry in the drying cabinet and sterilized in the oven at $160^{\circ} \mathrm{C}$ for two hours before use.

\section{$>$ Test organisms}

Tomato fruits purchased were left for one week for spoilage to occur. The spoilt tomato fruits were examined microbiologically using standard technique. Microorganisms isolated from these spoilt tomato fruits were stored on slants in the refrigerator.

\section{Total Bacterial and fungal load}

Total bacterial and fungal counts were determined using standard pour plate technique method. Nutrient agar and potato dextrose agar were used for isolating the bacteria and fungi respectively. Bacteria and fungi were identified using morphological and cultural characteristics as well as biochemical tests as described by APHA (2002) and Fawole and Oso (2004).

\section{$>$ Identification of bacteria isolates}

The identification of bacteria was based on morphological characteristics and biochemical tests which include Gram staining test, Motility test, Spore staining technique, Catalase test, Coagulase test, Methyl red test, Citrate test, Indole test, Oxidase test and Sugar fermentation according to methods of Fawole and Oso (2004). 
Identification of Fungal using Lactophenol Cotton Blue Mounts

Lactophenol cotton blue mounts was carried out on fungal isolates for full identification of the exact fungi based on the size, shape, surface feature of the conidia and hyphae arrangements. (Fawole and Oso, 2004).

\section{Pathogenicity test of the isolates}

Pathogenicity test in this study was carried out according to the methods of Chukwuka et al. (2010) and Onuorah et al.(2015).

\section{Drying and extraction of Trametes polyzona}

Trametes polyzona was collected, air dried and pulverised by an electrical mill. The powdered mushroom sample (100 g) was extracted with $2000 \mathrm{ml}$ of $95 \%$ ethanol, ethyl acetate, and n-hexane separately in an Erlenmeyer flask. The flasks were covered with aluminium foil and allowed to stand for 3 days for extraction with occasional stirring. The filtrate obtained was dried using rotary evaporator.

\section{Phytochemical screening of extracts}

The crude of Trametes polyzona was subjected to phytochemical screening for the presence of anthraquinones, alkaloids, tannins, saponins, phlobatannins, steroids, flavonoids and cardiac glycosides using standard techniques.

\section{Antimicrobial activity of extracts on Isolates}

Culture of the isolates maintained at $4^{\circ} \mathrm{C}$ on slopes of nutrient agar were used. Active cultures for experiments were prepared by transferring a loopful of cells from the stock cultures to testtubes of nutrient broth and were incubated without agitation for 24 hours at $36^{\circ} \mathrm{C}$.

\section{Shelf life determination}

Tomato fruits were divided into group A and B. Group A represent tomato fruits preserved with Trametes polyzona extract of water, ethanol, ethyl acetate and N-hexane, while group B were fruits preserved with only the solvent. Each of the group were subdivided into two; which contain tomato fruits kept in the refrigerator and fruits left at ambient temperature. The period of storage of the fruits so as to determine the shelf life was six weeks.

\section{$>$ Proximate Estimation}

The moisture, crude protein, crude fibre, crude fat, ash and soluble carbohydrate (by difference) were determined in accordance with AOAC (2012). The proximate analyses were carried out in triplicate.

\section{Vitamin $C$ determination}

Tomato juice extract in the range of $1,2,5,10$, and 15 $\mathrm{ml}$ were pipetted out into a $25 \mathrm{ml}$ standard flask followed by $2.1 \mathrm{ml}$ of standard potassium chromate, and $3 \mathrm{ml}$ of diphenylcarbazide solution. The mixture was diluted with $0.8 \mathrm{M}$ nitric acid up to the mark. (Prabha et al., 2011)

\section{Data Analysis}

All the experiments were carried out in triplicate and data obtained from the study were subjected to One-way analysis of variance (ANOVA) using SPSS version 23. Treatment means were compared using Duncan's New Multiple Range Test (DNMRT) at 5\% level of significance.

\section{RESULTS AND DISCUSION}

\section{Microbial load from spoilt tomato fruits}

Bacterial load of the spoilt tomato fruits ranged between $8.00 \times 10^{5}$ and $14.00 \times 10^{5} \mathrm{cfu} / \mathrm{g}$ while the fungal load ranged between $1.67 \times 10^{5}$ and $3.33 \times 10^{5} \mathrm{sfu} / \mathrm{g}$ as shown in Fig 1. Bacterial load is higher than fungal load in the tomato obtained from the three markets examined.

\section{Morphological and Biochemical Characteristics of Microbial load in tomato samples}

The cultural, morphological and biochemical characteristics of the bacterial and fungal isolates are shown in Tables 1, and 2 respectively. Eight bacteria and five fungi were isolated from the spoilt tomato fruits. They include; Staphylococcus aureus, Bacillus spp, Escherichia coli, Klebsiella spp, Lactobacillus spp, Shigella spp, Pseudomonas aeruginosa, Micrococcus spp, Rhizopus stolonifer, Aspergillus niger, A. terreus, A. flavus, and Fusarium spp.

\section{Prevalence of Microorganisms in the markets examined}

Occurrence of microorganisms isolated from the tomato samples shown in Table 3 revealed that Oja -oba Market and Isinkan Market had higher number of microbial isolates than Northgate Market. All the microbial isolates were present in market 2 except $A$. terreus. Escherichia coli and Fusarium spp were absent in market 2.

\section{Weight loss in tomato fruits after pathogenicity test}

Bacterial isolates recorded higher magnitude in weight loss of the tomato fruits than the fungal isolates as shown in Figs 2 and 3 respectively. All the microbial isolates showed significant effect on all the tomato samples. 


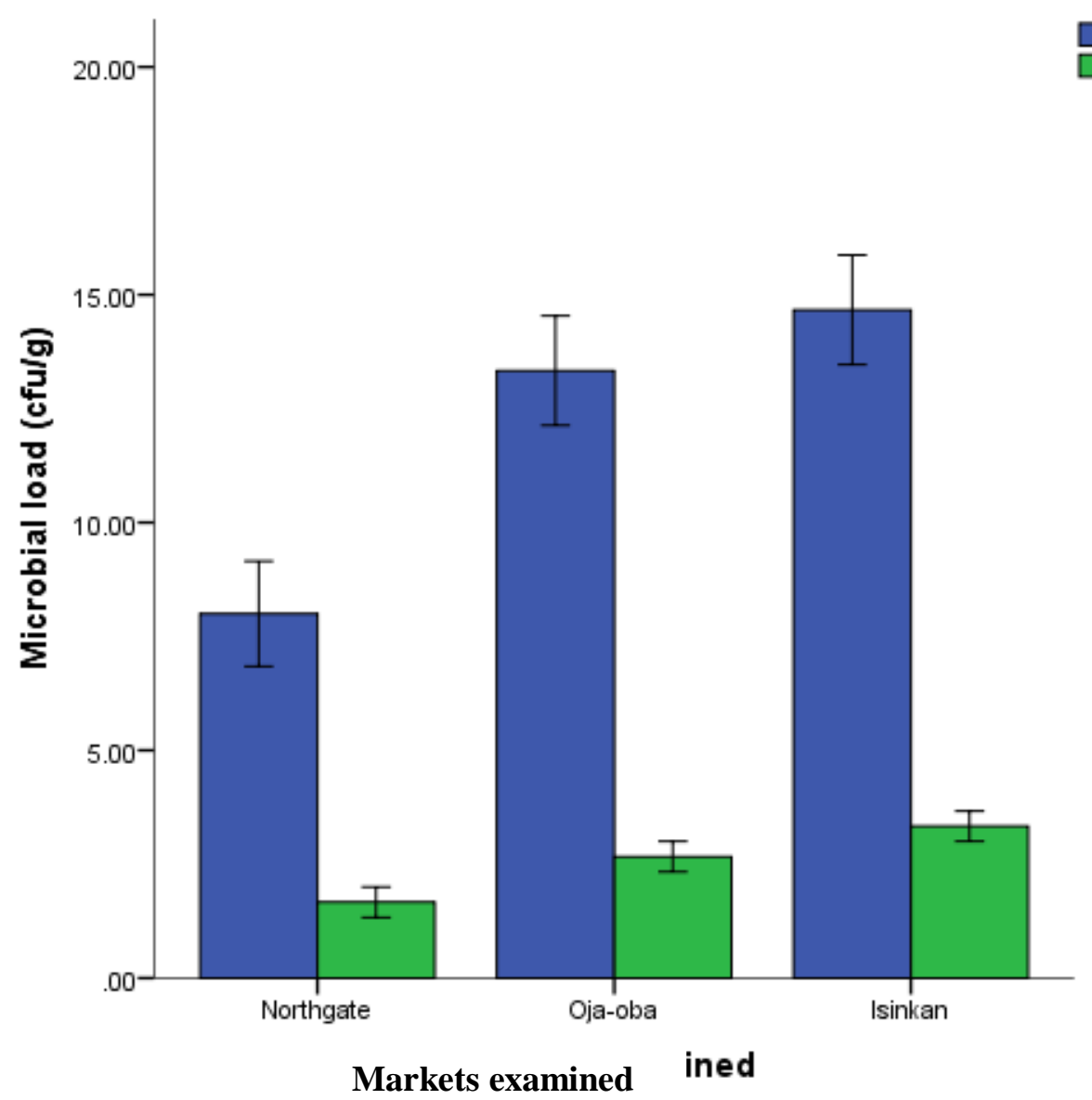

Total bacteria (cfu/ml)

Total fungi (sfu/g)

Fig 1:- Microbial load of spoilt Tomato fruits from different Markets

\begin{tabular}{|c|c|c|c|c|c|c|c|c|c|c|c|c|c|c|}
\hline \multirow{2}{*}{ Isolates } & \multicolumn{14}{|c|}{ Biochemical characteristics } \\
\hline & 舫 & ڤ̆ & 泀 & 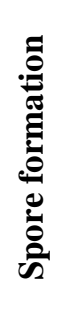 & 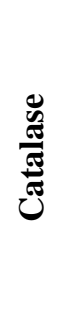 & 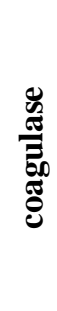 & & 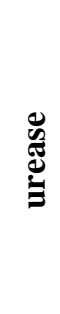 & $\frac{\stackrel{0}{\circ}}{\stackrel{\Xi}{g}}$ & 蒙 & 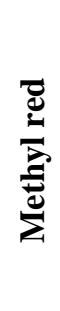 & 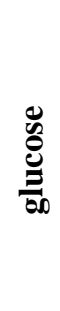 & 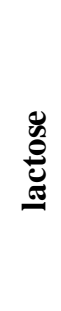 & 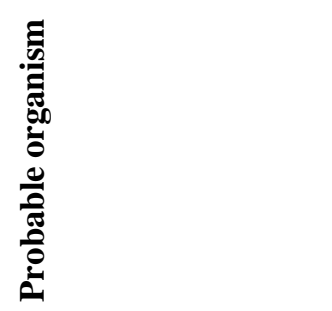 \\
\hline 1 & + & cocci & - & - & + & + & - & - & - & - & - & - & - & Staphylococcus aureus \\
\hline 2 & + & Rod & + & + & + & - & - & - & - & - & + & + & - & Bacillus spp \\
\hline 3 & - & Rod & - & - & - & - & + & - & + & - & + & + & - & Escherichia coli \\
\hline 4 & - & Rod & - & - & - & - & + & - & - & - & + & + & - & Klebsiella spp \\
\hline 5 & + & Rod & - & - & - & - & - & - & - & - & - & + & + & Lactobacillus spp \\
\hline 6 & - & Rod & + & - & - & - & - & - & - & - & - & + & - & Shigella spp \\
\hline 7 & - & Rod & - & - & - & - & - & - & - & + & - & + & - & Pseudomonas spp \\
\hline 8 & + & cocci & - & - & - & - & - & - & - & - & - & - & - & Micrococcus spp \\
\hline
\end{tabular}

Table 1:- Biochemical characteristics of bacterial isolates obtained from spoilt tomatoes and characteristics of bacterial isolate 
ISSN No:-2456-2165

\begin{tabular}{|c|c|c|}
\hline Isolate & Morphological Characteristics & Probable organism \\
\hline A & White cotton-like mycelia, non-septate hyphae, well developed collumela which is & Rhizopus stolonifer \\
in umbrella-like form. & Dark-brown conidia, long conidiophores, phialides are borne on brown metulae & Aspergilus niger \\
\hline B & $\begin{array}{c}\text { Whitish-pink mycelia, conidial heads are compact and biserate. Conidiophores are } \\
\text { smooth and hyaline. Aleurioconida produced directly on the hyphae }\end{array}$ & A.terreus \\
\hline D & Greenish mycelia, hyphae are septate, roughened and uncoloured conidiophores. & Alavus \\
\hline E & Reddish-white mycelia, hyphae are septate and hyaline. Phailades are cylindrical & Fusarium spp \\
\hline
\end{tabular}

Table 2:- Morphological characteristics of fungal isolates

\begin{tabular}{|c|c|c|c|}
\hline \multirow[t]{2}{*}{ Microbial isolates } & \multicolumn{3}{|c|}{ Markets examined } \\
\hline & $\mathrm{S} 1$ & $\mathrm{~S} 2$ & S3 \\
\hline Staphylococcus aureus & + & + & + \\
\hline Bacillus spp & + & + & + \\
\hline Escherichia coli & - & + & - \\
\hline Klebsiella spp & - & + & + \\
\hline Lactobacillus spp & + & + & + \\
\hline Shigella spp & + & + & + \\
\hline Pseudomonas spp & + & + & + \\
\hline Micrococcus spp & + & + & + \\
\hline Rhizopus stolonifer & + & + & + \\
\hline Aspergillus niger & + & + & + \\
\hline A. terreus & + & - & + \\
\hline A. flavus & - & + & + \\
\hline Fusarium spp & - & + & - \\
\hline
\end{tabular}

Table 3:- Occurrence of microorganisms in the Tomatoes obtained from different markets

Key: + = Present

- = Absent

S1 = Northgate Market
S2 = Oja Oba Market

S3 = Isinkan Market 


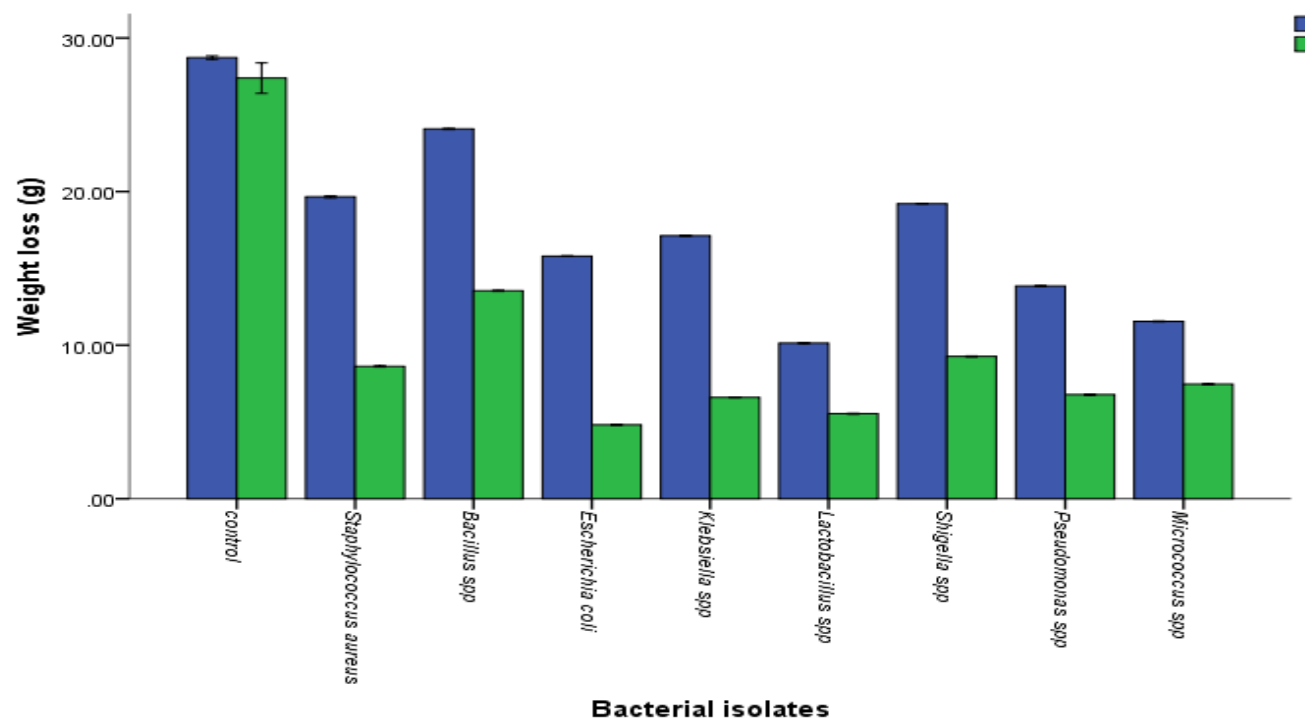

Fig 2: Weight of tomato fruits before and after pathogenicity test with bacterial isolates

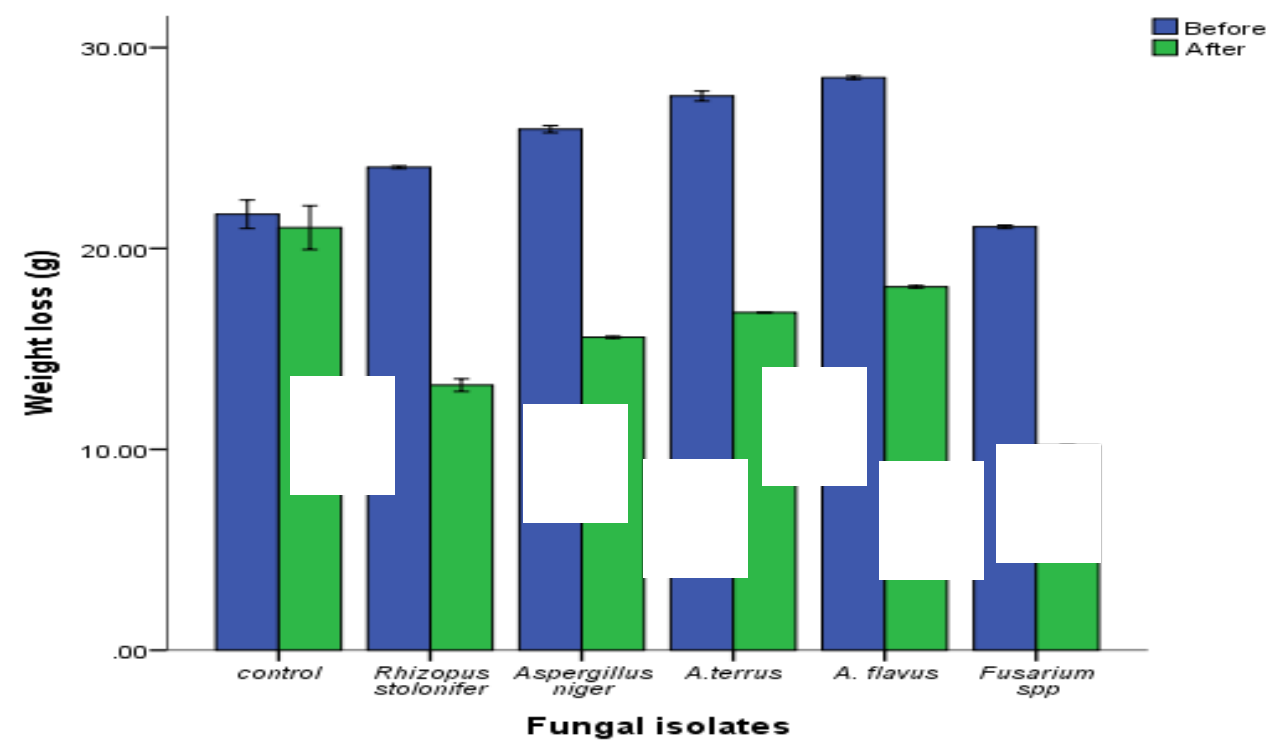

Fig 3:- Weight of tomato fruits before and after pathogenicity test with fungal isolates

\section{Qualitative phytochemical screening of extracts}

The qualitative analysis of the extract showed that saponin, tannin, flavonoid, and cardiac glycoside were present in all the extracts of Trametes polyzona while anthraquinone and phlobatannin were absent (Table 4).

\section{Quantitative phytochemical screening of extracts}

The quantitative analysis of the extracts showed variation in the phytochemical constituents of Trametes polyzona (Table 5).Ethanol and $\mathrm{N}$-hexane recorded significant content of saponin with value of $9.61 \mathrm{mg} / \mathrm{ml}$ and $7.92 \mathrm{mg} / \mathrm{ml}$ respectively.

\section{Antimicrobial activity of Trametes polyzona}

The extract exhibited varying antibacterial effect against all the selected isolates. N-hexane extract of
Trametes polyzona showed significant $(\mathrm{P}<0.05)$ inhibitory (for example Staphylococcus aureus $\left(10.55 \pm 0.05^{\mathrm{b}}\right) \mathrm{mm}$ while control has $\left(35.00 \pm 2.88^{\mathrm{e}}\right) \mathrm{mm}$ and all other bacteria isolated from the spoilt tomato fruits as shown in Table 6 . None of the extract showed inhibitory potential on the fungal isolates (Table 7).

Shelf life extension of Tomato fruits by Trametes polyzona extract

All the tomato fruits preserved with the extract and kept under refrigerator temperature were still looking wholesome in appearance, texture, colour and firmness after the fourth week of storage while tomato fruits preserved with only solvents showed signs of spoilage after the third week of storage as shown in Tables 7 and 8 respectively. 
ISSN No:-2456-2165

\begin{tabular}{|c|c|c|c|c|}
\hline Phytochemical component & Ethanol & Water & Ethyl acetate & + \\
\hline Saponin & + & + & + & + \\
\hline Tannin & + & + & + & + \\
\hline Phytate & + & + & + & + \\
\hline Flavonoid & + & + & + \\
\hline Terpenoid & + & + & + \\
\hline Total phenol & + & - & + \\
\hline Oxalate & - & - & - \\
\hline
\end{tabular}

Table 4:- Qualitative screening of Trametes polyzona

\begin{tabular}{|c|c|c|c|c|c|c|c|}
\hline \multirow{2}{*}{ Extract } & \multicolumn{7}{|c|}{ Phytochemical constituents } \\
\cline { 2 - 8 } & Saponin & Tannin & Phytate & Flavonoid & Terpenoid & Total phenol & oxalate \\
\hline ETHANOL & $9.61 \pm 0.07^{\mathrm{c}}$ & $2.12 \pm 0.02^{\mathrm{b}}$ & $0.57 \pm 0.02^{\mathrm{b}}$ & $0.90 \pm 0.03^{\mathrm{c}}$ & $0.27 \pm 0.01^{\mathrm{a}}$ & $0.93 \pm 0.02^{\mathrm{b}}$ & $0.04 \pm 0.00^{\mathrm{a}}$ \\
\hline WATER & $4.06 \pm 0.75^{\mathrm{a}}$ & $2.19 \pm 0.09^{\mathrm{b}}$ & $1.22 \pm 0.00^{\mathrm{c}}$ & $0.55 \pm 0.05^{\mathrm{b}}$ & $0.18 \pm 0.00^{\mathrm{a}}$ & $1.78 \pm 0.09^{\mathrm{c}}$ & $0.03 \pm 0.00^{\mathrm{a}}$ \\
\hline ETHYL- & $5.14 \pm 0.33^{\mathrm{a}}$ & $0.90 \pm 0.00^{\mathrm{a}}$ & $0.46 \pm 0.01^{\mathrm{a}}$ & $3.69 \pm 0.16^{\mathrm{d}}$ & $1.45 \pm 0.02^{\mathrm{b}}$ & $0.07 \pm 0.01^{\mathrm{a}}$ & $0.07 \pm 0.01^{\mathrm{b}}$ \\
\hline ACETATE & & & & & & & \\
\hline N-HEXANE & $7.92 \pm 0.33^{\mathrm{b}}$ & $0.76 \pm 0.03^{\mathrm{a}}$ & $0.44 \pm 0.01^{\mathrm{a}}$ & $0.21 \pm 0.01^{\mathrm{a}}$ & $2.96 \pm 0.34^{\mathrm{c}}$ & $0.21 \pm 0.01^{\mathrm{a}}$ & $0.03 \pm 0.00^{\mathrm{a}}$ \\
\hline
\end{tabular}

Table 5:- Quantitative Phytochemical Constituents $(\mathrm{mg} / \mathrm{g})$ of Trametes polyzonaextracts

Data are presented as Mean \pm S.E $(n=3)$. Values with the same superscript letter(s) along the same column are not significantly different $(\mathrm{P}<0.05)$

\begin{tabular}{|c|c|c|c|c|c|c|c|c|}
\hline \multirow[b]{2}{*}{ Extract } & \multicolumn{8}{|c|}{ Zones of inhibition (mm) } \\
\hline & $\begin{array}{l}\text { Staphylococ } \\
\text { cus aureus }\end{array}$ & $\begin{array}{c}\text { Bacillus } \\
\text { spp }\end{array}$ & $\begin{array}{l}\text { Escherichi } \\
\text { a coli }\end{array}$ & $\begin{array}{l}\text { Klebsiella } \\
\text { spp }\end{array}$ & $\begin{array}{c}\text { Lactobacill } \\
\text { us spp }\end{array}$ & $\begin{array}{c}\text { Shigella } \\
\text { spp }\end{array}$ & $\begin{array}{c}\text { Pseudomon } \\
\text { as spp }\end{array}$ & $\begin{array}{c}\text { Micrococc } \\
\text { us spp }\end{array}$ \\
\hline ETHANOL & $28.16 \pm 1.30^{\mathrm{d}}$ & $2.51 \pm 0.01^{\mathrm{b}}$ & $0.00 \pm 0.00^{\mathrm{a}}$ & $3.13 \pm 0.57^{\mathrm{b}}$ & $0.00 \pm 0.00^{\mathrm{a}}$ & $0.00 \pm 0.00^{\mathrm{a}}$ & $0.00 \pm 0.00^{\mathrm{a}}$ & $5.65 \pm 0.05^{\mathrm{b}}$ \\
\hline WATER & $5.23 \pm 0.03^{\mathrm{a}}$ & $0.00 \pm 0.00^{\mathrm{a}}$ & $2.30 \pm 0.03^{\mathrm{b}}$ & $0.00 \pm 0.00^{\mathrm{a}}$ & $2.02 \pm 0.02^{\mathrm{b}}$ & $0.00 \pm 0.00^{\mathrm{a}}$ & $0.00 \pm 0.00^{\mathrm{a}}$ & $0.00 \pm 0.00^{\mathrm{a}}$ \\
\hline $\begin{array}{l}\text { ETHYL- } \\
\text { ACETAE }\end{array}$ & $12.65 \pm 0.58^{c}$ & $5.35 \pm 0.55^{\mathrm{c}}$ & $4.21 \pm 0.01^{\mathrm{c}}$ & $6.07 \pm 0.07^{\mathrm{c}}$ & $6.31 \pm 0.06^{\mathrm{c}}$ & $\begin{array}{c}10.02 \pm 0.0 \\
2^{\mathrm{b}}\end{array}$ & $0.00 \pm 0.00^{\mathrm{a}}$ & $5.00 \pm 0.00^{\mathrm{b}}$ \\
\hline $\begin{array}{c}\mathrm{N}- \\
\text { HEXANE }\end{array}$ & $10.55 \pm 0.05^{b}$ & $\begin{array}{c}27.00 \pm 1.5 \\
2^{\mathrm{d}}\end{array}$ & $\begin{array}{c}22.66 \pm 1.2 \\
0^{d}\end{array}$ & $2.04 \pm 0.04^{\mathrm{b}}$ & $14.00 \pm 0.58^{d}$ & $\begin{array}{c}11.66 \pm 0.6 \\
6^{\mathrm{b}}\end{array}$ & $17.66 \pm 1.20^{\mathrm{b}}$ & $\underset{c}{22.66 \pm 1.20}$ \\
\hline CIPROTAB & $35.00 \pm 2.88^{\mathrm{e}}$ & $\begin{array}{c}26.33 \pm 1.8 \\
5^{\mathrm{d}}\end{array}$ & $\begin{array}{c}23.33 \pm 1.2 \\
0^{\mathrm{d}}\end{array}$ & $\begin{array}{c}21.45 \pm 0.0 \\
5^{\mathrm{d}}\end{array}$ & $22.66 \pm 1.20^{\mathrm{e}}$ & $\begin{array}{c}25.66 \pm 1.2 \\
0^{c}\end{array}$ & $21.66 \pm 1.76^{\mathrm{c}}$ & $\underset{c}{23.66 \pm 0.88}$ \\
\hline
\end{tabular}

Table 6:- Antibacterial properties of extracts of Trametes polyzona

Data are presented as Mean \pm S.E (n=3). Values with the same superscript letter(s) along the same column are not significantly different $(\mathrm{P}<0.05)$.

\begin{tabular}{|c|c|c|c|c|c|}
\hline \multirow{2}{*}{ Extract } & \multicolumn{4}{|c|}{ Zones of inhibition (mm) } \\
\cline { 2 - 5 } & $\begin{array}{c}\text { Rhizopus } \\
\text { stolonifer }\end{array}$ & Aspergillus niger & A.terrus & A.flavus & \multirow{2}{*}{ Fusarium spp } \\
\hline ETHANOL & $0.00 \pm 0.00^{\mathrm{a}}$ & $0.00 \pm 0.00^{\mathrm{a}}$ & $0.00 \pm 0.00^{\mathrm{a}}$ & $0.00 \pm 0.00^{\mathrm{a}}$ & $0.00 \pm 0.00^{\mathrm{a}}$ \\
\hline WATER & $0.00 \pm 0.00^{\mathrm{a}}$ & $0.00 \pm 0.00^{\mathrm{a}}$ & $0.00 \pm 0.00^{\mathrm{a}}$ & $0.00^{\mathrm{a}}$ & $0.00 \pm 0.00^{\mathrm{a}}$ \\
\hline ETHYL-ACETATE & $0.00 \pm 0.00^{\mathrm{a}}$ & $0.00 \pm 0.00^{\mathrm{a}}$ & $0.00 \pm 0.00^{\mathrm{a}}$ & $0.00 \pm 0.00^{\mathrm{a}}$ & $0.00 \pm 0.00^{\mathrm{a}}$ \\
\hline N-HEXANE & $0.00 \pm 0.00^{\mathrm{a}}$ & $0.00 \pm 0.00^{\mathrm{a}}$ & $0.00 \pm 0.00^{\mathrm{a}}$ & $0.00 \pm 0.00^{\mathrm{a}}$ & $0.00 \pm 0.00^{\mathrm{a}}$ \\
\hline Griseofluvin & $15.00 \pm 1.88^{\mathrm{b}}$ & $14.33 \pm 1.85^{\mathrm{b}}$ & $12.03 \pm 1.03^{\mathrm{b}}$ & $15.45 \pm 0.05^{\mathrm{b}}$ & $12.66 \pm 1.20^{\mathrm{b}}$ \\
\hline
\end{tabular}

Table 7:- Antifungal properties of extracts of Trametes polyzona

Data are presented as Mean \pm S.E ( $n=3)$. Values with the same superscript letter(s) along the same column are not significantly different $(\mathrm{P}<0.05)$ 
ISSN No:-2456-2165

\begin{tabular}{|c|c|c|c|c|c|c|c|c|c|c|c|c|c|c|c|c|c|c|c|c|c|c|c|c|c|}
\hline \multirow{3}{*}{$\begin{array}{l}\text { Weeks } \\
\text { observed }\end{array}$} & \multirow{3}{*}{ Extract } & \multicolumn{6}{|c|}{ Appearance } & \multicolumn{6}{|c|}{ Texture } & \multicolumn{6}{|c|}{ Color } & \multicolumn{6}{|c|}{ Firmness } \\
\hline & & \multicolumn{3}{|c|}{ Amb } & \multicolumn{3}{|c|}{ Ref } & \multicolumn{3}{|c|}{ Amb } & \multicolumn{3}{|c|}{ Ref } & \multicolumn{3}{|c|}{$\mathrm{Amb}$} & \multicolumn{3}{|l|}{ Ref } & \multicolumn{3}{|c|}{ Amb } & \multicolumn{3}{|c|}{ Ref } \\
\hline & & S1 & S2 & S3 & S1 & S2 & S3 & S1 & S2 & S3 & S1 & $\mathrm{S} 2$ & S3 & S1 & S2 & S3 & S1 & S2 & S3 & S1 & S2 & $\mathrm{S} 3$ & S1 & $\mathrm{S} 2$ & S3 \\
\hline & Water & ++ & ++ & ++ & + & ++ & ++ & ++ & ++ & ++ & ++ & ++ & ++ & ++ & ++ & ++ & ++ & ++ & ++ & ++ & ++ & ++ & ++ & ++ & ++ \\
\hline \multirow[t]{4}{*}{ Week 1} & Ethanol & + & H & ++ & + & H & + & + & ++ & H & H & + & H & H & + & + & ++ & H & H & + & + & + & ++ & + & ++ \\
\hline & Ethyl acetate & ++ & ++ & ++ & ++ & ++ & ++ & ++ & ++ & ++ & ++ & ++ & ++ & ++ & ++ & ++ & ++ & ++ & ++ & ++ & ++ & ++ & ++ & ++ & ++ \\
\hline & N-hexane & + & + & + & + & + & ++ & + & ++ & ++ & + & ++ & + & ++ & ++ & ++ & ++ & ++ & + & + & + & + & ++ & + & ++ \\
\hline & Water & ++ & ++ & ++ & ++ & ++ & ++ & ++ & ++ & ++ & + & ++ & + & ++ & ++ & ++ & ++ & ++ & + & ++ & + & ++ & ++ & ++ & ++ \\
\hline \multirow[t]{4}{*}{ Week 2} & Ethanol & & + & + & + & + & ++ & + & + & + & ++ & ++ & + & + & + & + & ++ & ++ & ++ & + & + & + & ++ & ++ & ++ \\
\hline & Ethyl acetate & + & + & + & ++ & ++ & ++ & + & + & + & ++ & ++ & ++ & + & + & + & ++ & ++ & + & + & + & + & ++ & ++ & ++ \\
\hline & N-hexane & + & + & + & ++ & ++ & ++ & + & + & + & ++ & ++ & ++ & + & + & + & ++ & ++ & ++ & + & + & + & ++ & ++ & ++ \\
\hline & Water & + & + & + & + & + & + & + & + & + & + & + & + & + & + & + & + & + & + & + & + & + & + & + & + \\
\hline \multirow[t]{3}{*}{ Week 3} & Ethanol & - & - & & ++ & ++ & ++ & - & - & - & ++ & ++ & ++ & + & + & + & ++ & ++ & ++ & + & + & + & ++ & ++ & ++ \\
\hline & Ethyl acetate & - & - & - & ++ & ++ & ++ & - & - & - & ++ & ++ & ++ & + & - & - & ++ & ++ & + & - & - & + & ++ & ++ & ++ \\
\hline & N-hexane & + & + & + & ++ & ++ & ++ & + & + & + & ++ & ++ & ++ & + & + & + & + & ++ & + & - & - & - & ++ & ++ & + \\
\hline \multirow[t]{5}{*}{ Week 4} & Water & - & - & - & + & + & + & - & - & - & + & + & + & - & - & - & + & + & + & - & - & - & + & + & + \\
\hline & Ethanol & - & - & - & + & + & + & - & - & - & + & + & + & + & + & + & + & + & + & + & + & + & + & + & + \\
\hline & Ethyl acetate & - & - & - & + & + & + & - & - & - & + & + & + & + & - & - & + & + & + & - & - & + & + & + & + \\
\hline & N-hexane & - & - & - & + & + & + & + & + & + & + & + & + & + & + & + & + & + & + & - & - & - & + & + & + \\
\hline & Water & - & - & - & - & - & - & - & - & - & - & - & - & - & - & - & - & - & - & - & - & - & - & - & - \\
\hline \multirow[t]{4}{*}{ Week 5} & Ethanol & - & - & - & + & + & + & - & - & - & + & + & + & + & + & + & + & + & + & + & + & + & + & + & + \\
\hline & Ethyl acetat & - & - & - & + & + & + & - & - & - & - & - & - & - & - & - & + & + & + & - & - & - & - & - & - \\
\hline & N-hexane & - & - & - & + & + & + & - & - & - & + & + & + & - & - & - & + & + & + & - & - & - & + & + & + \\
\hline & Water & - & - & - & - & - & - & - & - & - & - & - & - & - & - & - & - & - & - & - & - & - & - & - & - \\
\hline \multirow[t]{3}{*}{ Week 6} & Ethanol & - & - & - & - & - & - & - & - & - & + & + & + & - & - & - & - & - & - & - & - & - & + & + & + \\
\hline & Ethyl acetate & - & - & - & - & - & - & - & - & - & - & - & - & - & - & - & - & - & - & - & - & - & - & - & - \\
\hline & N-hexane & - & - & - & + & + & + & - & - & - & + & + & + & - & - & - & + & + & + & - & - & - & - & - & - \\
\hline
\end{tabular}

Table 8:- Sensory properties of Tomato fruits during preservation with extracts of Trametes polyzona

Key: Amb- Ambient temperature, Ref- Refrigeration, S1- Northgate market, S2- Oja-oba market, S3- Isinkan market, (++) = Very good, $(+)=$ Good, $(-)=$ Bad

\begin{tabular}{|c|c|c|c|c|c|c|c|c|c|c|c|c|c|c|c|c|c|c|c|c|c|c|c|c|c|}
\hline \multirow{3}{*}{$\begin{array}{c}\text { Weeks } \\
\text { observed }\end{array}$} & \multirow[t]{3}{*}{ Control } & \multicolumn{6}{|c|}{ Appearance } & \multicolumn{6}{|c|}{ Texture } & \multicolumn{6}{|c|}{ Color } & \multicolumn{6}{|c|}{ Firmness } \\
\hline & & \multicolumn{3}{|c|}{$\mathrm{Amb}$} & \multicolumn{3}{|c|}{ Ref } & \multicolumn{3}{|c|}{$\mathrm{Amb}$} & \multicolumn{3}{|c|}{ Ref } & \multicolumn{3}{|c|}{ Amb } & \multicolumn{3}{|c|}{ Ref } & \multicolumn{3}{|c|}{ Amb } & \multicolumn{3}{|c|}{ Ref } \\
\hline & & S1 & S2 & S3 & S1 & S2 & S3 & S1 & S2 & S3 & S1 & S2 & S3 & S1 & S2 & S3 & S1 & S2 & S3 & S1 & S2 & S3 & S1 & S2 & S3 \\
\hline & Water & ++ & + & + & + & + & + & ++ & + & + & + & + & ++ & + & + & + & + & + & + & + & + & + & + & + & + \\
\hline \multirow[t]{4}{*}{ Week l } & Ethanol & + & + & + & + & + & + & ++ & + & + & ++ & + & ++ & + & + & + & + & ++ & + & + & + & ++ & + & + & + \\
\hline & Ethyl acetate & + & + & + & + & + & + & + & + & + & + & + & + & + & + & + & + & + & + & + & + & + & + & + & + \\
\hline & N-hexane & - & - & - & + & + & + & - & - & - & + & + & + & - & - & - & + & + & + & - & - & - & + & ++ & + \\
\hline & Water & - & - & - & + & + & + & - & - & - & + & + & + & - & - & - & + & + & + & - & - & - & + & + & + \\
\hline \multirow[t]{4}{*}{ Week 2} & Ethanol & + & + & + & ++ & ++ & + & + & + & + & + & ++ & + & + & + & + & ++ & ++ & + & + & + & + & ++ & ++ & + \\
\hline & Ethyl acetate & + & + & + & + & ++ & ++ & + & + & + & + & ++ & + & + & + & + & ++ & ++ & + & + & + & + & ++ & ++ & + \\
\hline & N-hexane & - & - & - & + & ++ & + & - & - & - & + & ++ & ++ & - & - & - & + & ++ & ++ & - & - & - & ++ & ++ & + \\
\hline & Water & - & - & - & + & + & + & - & - & - & + & + & + & - & - & - & + & + & + & - & - & - & + & + & + \\
\hline \multirow[t]{3}{*}{ Week 3} & Ethanol & + & + & + & + & + & + & + & + & + & + & + & + & + & + & + & + & + & + & + & + & + & + & + & + \\
\hline & Ethyl acetate & - & - & - & + & + & + & + & + & + & + & + & + & + & + & + & + & + & + & + & + & + & + & + & + \\
\hline & N-hexane & - & - & - & + & + & + & - & - & - & + & + & + & - & - & - & + & + & + & - & - & - & + & + & + \\
\hline \multirow[t]{5}{*}{ Week 4} & Water & - & - & - & + & + & + & - & - & - & + & + & + & - & - & - & + & + & + & - & - & - & - & - & - \\
\hline & Ethanol & - & - & - & + & + & + & - & - & - & + & + & + & - & - & - & + & + & + & - & - & - & + & + & + \\
\hline & Ethyl acetate & - & - & - & + & + & + & - & - & - & + & + & + & - & - & - & + & + & + & - & - & - & + & + & + \\
\hline & N-hexane & - & - & - & + & + & + & - & - & - & + & + & + & - & - & - & + & + & + & - & - & - & + & + & + \\
\hline & Water & - & - & - & - & - & - & - & - & - & - & - & - & - & - & - & - & - & - & - & - & - & - & - & - \\
\hline \multirow[t]{4}{*}{ Week 5} & Ethanol & - & - & - & - & - & - & - & - & - & - & - & - & - & - & - & - & - & - & - & - & - & - & - & - \\
\hline & Ethyl acetate & - & - & - & - & - & - & - & - & - & - & - & - & - & - & - & - & - & - & - & - & - & - & - & - \\
\hline & N-hexane & - & - & - & - & - & - & - & - & - & - & - & - & - & - & - & & - & - & - & - & - & - & - & - \\
\hline & Water & - & - & - & - & - & - & - & - & - & - & - & - & - & - & - & - & - & - & - & - & - & - & - & - \\
\hline \multirow[t]{3}{*}{ Week 6} & Ethanol & - & - & - & - & - & - & - & - & - & - & - & - & - & - & - & - & - & - & - & - & - & - & - & - \\
\hline & Ethyl acetate & - & - & - & - & - & - & - & - & - & - & - & - & - & - & - & - & - & - & - & - & - & - & - & - \\
\hline & N-hexane & - & - & - & - & - & - & - & - & - & - & - & - & - & - & - & & - & - & - & - & - & - & - & - \\
\hline
\end{tabular}

Table 9:- Sensory properties of Tomato fruits during preservation with the control (only the solvents)

Key: Amb- Ambient temperature, Ref- Refrigeration, S1- Northgate market, S2- Oja-oba market, S3- Isinkan market, (++) - Very good, (+) -Good, (-) - Bad 


\section{Proximate composition of fresh and preserved tomato fruits}

Tomato fruits preserved with Trametes polyzona extractrecorded higher proximate composition than the fresh fruits as revealed in Table 9. Tomato fruit preserved with N-hexane extract of Trametes polyzona had significant protein and carbohydrate content with value of $3.26 \%$ and $15.33 \%$ respectively.

\section{Vitamin C content of tomato fruits}

Tomato fruits preserved with ethyl acetate recorded the highest vitamin $\mathrm{C}$ content as shown in Fig 4, followed by tomato fruits preserved with ethanol.

\begin{tabular}{|c|c|c|c|c|c|c|c|}
\hline \multirow{2}{*}{} & \multirow{2}{*}{ Samples } & \multicolumn{6}{|c|}{ Proximate composition (\%) } \\
\cline { 3 - 7 } & & Ash & Moisture & Fibre & Fat & Protein & Carbohydrate \\
\cline { 3 - 7 } & S1 & $0.41 \pm 0.00^{\mathrm{c}}$ & $63.02 \pm 0.03^{\mathrm{cd}}$ & $23.91 \pm 0.00^{\mathrm{c}}$ & $6.03 \pm 0.30^{\mathrm{b}}$ & $0.67 \pm 0.00^{\mathrm{c}}$ & $5.94 \pm 0.27^{\mathrm{b}}$ \\
\hline & $\mathrm{S} 2$ & $0.36 \pm 0.00^{\mathrm{b}}$ & $63.82 \pm 0.57^{\mathrm{d}}$ & $20.83 \pm 0.00^{\mathrm{a}}$ & $5.85 \pm 0.41^{\mathrm{b}}$ & $0.63 \pm 0.00^{\mathrm{a}}$ & $8.47 \pm 0.15^{\mathrm{c}}$ \\
\hline Fresh & S3 & $0.31 \pm 0.00^{\mathrm{a}}$ & $62.02 \pm 0.64^{\mathrm{c}}$ & $21.94 \pm 0.00^{\mathrm{b}}$ & $11.97 \pm 0.19^{\mathrm{d}}$ & $0.66 \pm 0.01^{\mathrm{b}}$ & $3.08 \pm 0.84^{\mathrm{a}}$ \\
\hline $\begin{array}{c}\text { Treated and } \\
\text { Stored }\end{array}$ & Ethanol & $1.01 \pm 0.00^{\mathrm{c}}$ & $53.50 \pm 0.00^{\mathrm{b}}$ & $28.23 \pm 0.00^{\mathrm{f}}$ & $6.19 \pm 0.01^{\mathrm{b}}$ & $3.22 \pm 0.00^{\mathrm{e}}$ & $8.05 \pm 0.21^{\mathrm{c}}$ \\
\hline & Water & $0.44 \pm 0.02^{\mathrm{c}}$ & $58.02 \pm 0.02^{\mathrm{bc}}$ & $23.91 \pm 0.05^{\mathrm{c}}$ & $9.07 \pm 0.07^{\mathrm{d}}$ & $1.51 \pm 0.00^{\mathrm{ab}}$ & $8.47 \pm 0.15^{\mathrm{c}}$ \\
\hline & $\begin{array}{c}\text { Ethyl } \\
\text { acetate }\end{array}$ & $1.11 \pm 0.00^{\mathrm{d}}$ & $52.45 \pm 0.00^{\mathrm{ab}}$ & $26.76 \pm 0.00^{\mathrm{e}}$ & $8.30 \pm 0.00^{\mathrm{c}}$ & $2.51 \pm 0.00^{\mathrm{d}}$ & $8.83 \pm 0.00^{\mathrm{c}}$ \\
& & & & & $3.26 \pm 0.00^{\mathrm{f}}$ & $15.53 \pm 0.03^{\mathrm{d}}$ \\
\hline
\end{tabular}

Table 10: Proximate composition (\%)of fresh and stored tomatoes

Data are presented as Mean \pm S.E (n=3). Values with the same superscript letter(s) along the same column are not significantly different $(\mathrm{P}<0.05)$

S1- Northgate market, S2- Oja-oba market, S3- Isinkan market

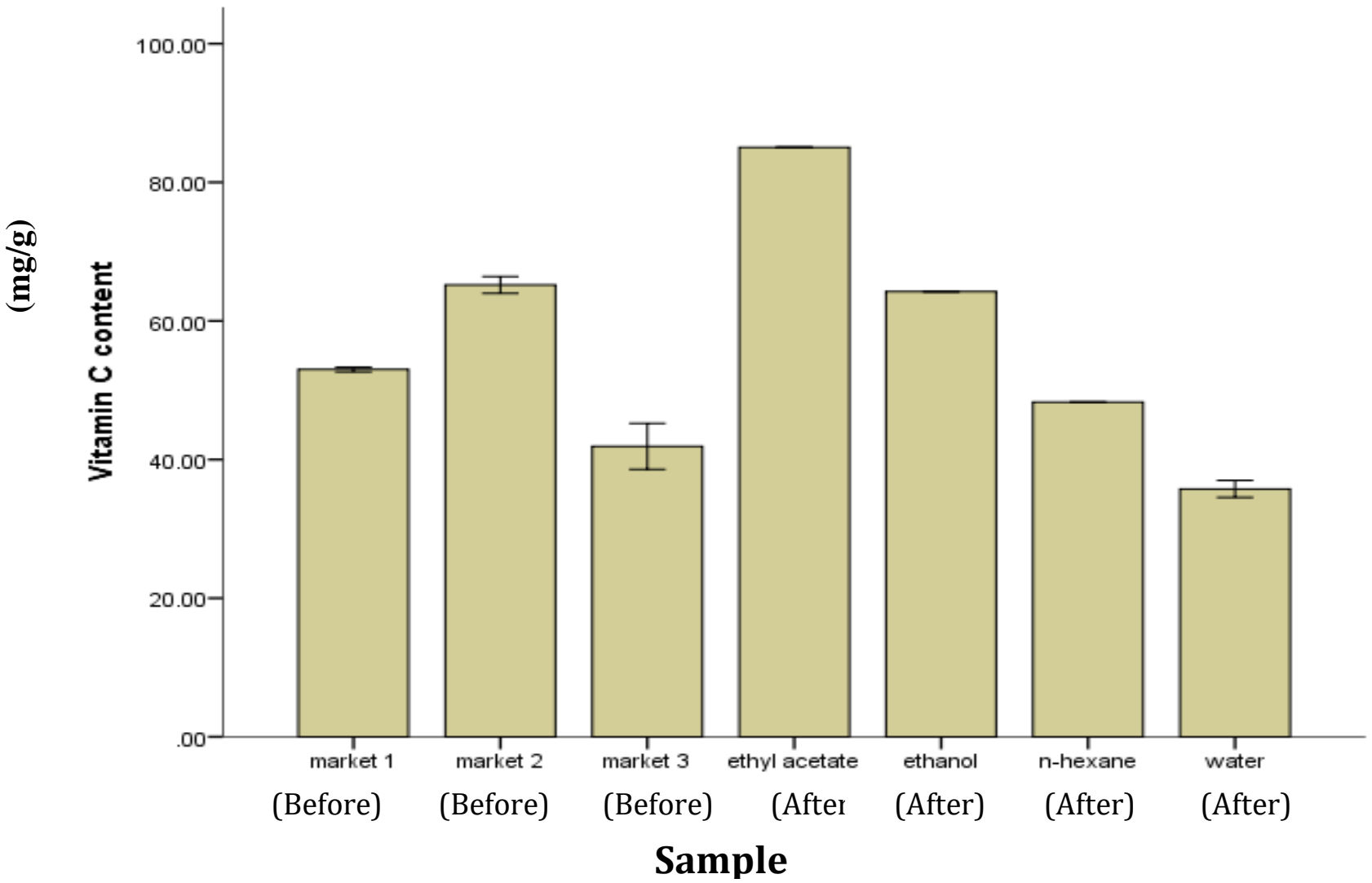

Fig 4:- Vitamin C content of tomato fruits before and after preservation 
Microbial load of spoilt tomato fruits showed that bacterial load is higher than fungal load in all the markets examined. Bacterial spoilage of tomatoes may also be attributable to the high water content, environmental conditions, state of handling, state of storage facilities, the microbial load of the handlers and the quality of the tomatoes (Samuel and Orji, 2015).Report on microbial load is comparable to Agbabiaka et al. (2015) during microbial examination of tomato fruits from different markets.

A total number of eight bacteria; Staphylococcus aureus, Bacillus spp, Escherichia coli, Klebsiella spp, Lactobacillus spp, Shigella spp, Pseudomonas aeruginosa, and Micrococcus spp, were isolated from the tomato fruits. While the five fungi isolated include; Rhizopus stolonifer, Aspergillus niger, A. terreus, A. flavus, and Fusarium spp. Similar microorganisms were also reported by Oyemaechi et al. (2014), during the microbial examination of spoilt tomato fruits in Onitsha metropolis. Report on the occurrence of microorganisms showed that Staphylococcus aureus, Bacillus spp, Lactobacillus spp, Shigella spp, $P$. aeruginosa, Micrococcus spp, Rhizopus stolonifera, and A.niger were found on tomato fruits collected from all the markets studied. The presence of Bacillus spp, Staphylococcus aureus, Rhizopus stolonifer and Aspergillus spp in the tomato fruits agreed with the findings of Bello et al. (2016), who also reported that these microorganisms are responsible for the spoilage of tomato fruits. Fajola (2006) reported that Rhizopus stolonifer is a soft rot pathogen of tomato in Nigeria. The mycelium of the fungus can infect adjacent fruits, through mechanical wounds and natural openings, creating a nest of mold growth on diseased fruits (Mahovic et al., 2009).

Bartz et al. (2009) also reported that certain species of Pseudomonas and Bacillus can cause soft rot of tomato fruits. Similarly, the presence of enteric organisms such as E.coli, Klebsiella spp, and Shigella spp in the spoilt tomato fruits is an indication that the tomato fruits were exposed to faecal contaminated water or organic manure. Many researchers had reported isolation of similar microorganisms from tomato fruits (Chuku et al., 2008; Akinmusire, 2011; Ibrahim et al., 2011; Chinedu and Enya, 2014; Wogu and Ofuase, 2014).

It was observed from this study that tomato fruits collected from Oja-oba Market and Isinkan Market had higher number of microbial isolates than Northgate Market. This indicated that tomato fruits sold at Oja-oba Market and Isinkan Market may have not been handled well during transportation and sales than those sold at Northgate Market. This report on the occurrence of microorganisms on tomato fruits agreed with the findings of Bello et al. (2016), who reported variation in the occurrence of microorganisms on tomato fruits collected from three different markets.
The result of the pathogenicity test of all the microbial isolates on tomato fruit samples after one week of inoculation showed significant $(\mathrm{P}>0.05)$ weight loss with bacterial isolates recording higher magnitude compared with fungi. Changes like wrinkled textures on the fruits and weight lost were observed after one week of inoculation of the spoilage microorganisms.

The Antibacterial assay revealed that N-hexane extract of Trametes polyzona showed significant $(\mathrm{P}>0.05)$ inhibitory effect on all the bacteria isolated from the spoilt tomato fruits. Ethyl-acetate extract of Trametes polyzona showed inhibitory effect on all the bacterial isolates except Pseudomonas aeruginosa. Ethanol extract only showed significant $(\mathrm{P}>0.05)$ inhibitory effect on Staphylococcus aureus. None of the extracts showed inhibitory potential on the fungal isolates.

The result from the Shelf life assessment showed that tomato fruits preserved with the extracts and kept in the refrigerator were acceptable in appearance, texture, color and firmness after the fourth week of storage. N-hexane and ethanol extract of the mushroom were observed to retain the texture of the tomato fruits kept in refrigerator even at the sixth week of storage. The shelf life potential of Trametes polyzona extract could be attributed to the presence of phenolic compounds in the extract which have been reported to exhibit an excellent preservative potential on food products containing lipids (Soto et al., 2011; Abugria and McElhenney, 2013). Thus, tomato fruits preserved with Trametes polyzona extract showed extended shelf life than those preserved with only solvent. Tomato fruits kept in refrigerator temperature also had a longer shelf life than those kept in ambient temperature. It has been reported to play a key role in minimizing food spoilage and waste (James et al., 2017). Consequently, tomato fruits preserved with Trametes polyzona extract and kept in the refrigerator had longer shelf life when compared with fruits kept in ambient temperature even though preserved with Trametes polyzona extract.

Proximate analysis revealed that moisture content of preserved tomato fruits were lower than fresh tomato fruits. Reduction in the moisture content of the preserved tomato fruits makes them less susceptible to spoilage by the action of microorganisms thus, increasing the shelf life of the tomato fruits (Samuel and Orji, 2015). The high moisture content of the fresh tomato fruits provides an enabling environment for proliferation of the microorganisms and hence its spoilage and potential to become health risk to human beings (Agbabiaka et al., 2015). Ash and fibre content of the preserved tomato fruits was recorded to be higher than fresh tomato fruits. The ash content is a measure/reflection of the nutritionally important mineral contents present in the food material (Omotosho 2005; Nnamani et al. 2009). Therefore, the high ash content recorded in tomato fruits preserved with Trametes polyzona extracts indicates that the fruits would provide essential valuable and useful minerals needed for body development (Gemede et al., 2015). 
Dietary fiber promotes the growth and protects the beneficial intestinal flora. Moreover, high intake of fiber reduces the risk of colon cancer (Dawczynski et al. 2007). Fat content of tomato fruits preserved with ethyl acetate extract of Trametes polyzona was observed to be higher than other extracts. Fat in diet also serve as a source of energy to man (Olaniyi et al., 2010). Protein and carbohydrate content of the preserved tomato fruits was higher than fresh tomato fruits. Although tomato fruits had been reported to be rich in vitamins and minerals. (Oyemaechi et al., 2014). Also, tomato fruits preserved with ethyl acetate extract of Trametes polyzona had the highest Vitamin C content of all tomato fruits examined. Vitamin $\mathrm{C}$ also known as ascorbic acid, is a dietary antioxidants which help to reduce the impact of oxidative stress in human (Giovannucci, 1999; Matteo et al., 2010).

\section{CONCLUSION}

In conclusion, frequent inspection of the fruits for sale by food inspectors is also recommended, so as to prevent the consumption of contaminated tomato fruits thereby reducing the health hazards posed by some of the opportunistic toxinproducing microorganisms isolated in this study. Trametes polyzona extract showed antimicrobial potential which consequently increased the shelf life of the tomato fruits. More so, the nutrient content of tomato fruits preserved with The overall result revealed that Staphylococcusaureus, Bacillus spp, Lactobacillus spp, Shigella spp, Pseudomonas aeruginosa, Micrococcus spp, Rhizopus stolonifera, and Aspergillus niger were the dominant microorganisms isolated from the tomato fruits. N-hexane extract of Trametes polyzona showed inhibitory effect on all the bacterial isolates, while Ethyl-acetate extract of Trametes polyzona showed inhibitory effect on all the bacterial isolates except Pseudomonas aeruginosa. All the tomato fruits preserved with the extracts and kept in refrigerator temperature were acceptable in appearance, texture, and colour after the fourth week of storage. N-hexane and ethanol extract of the mushroom were observed to preserve the texture of the tomato fruits kept in refrigerator even at the sixth week of storage. Proximate analysis revealed that moisture content of preserved tomato fruits were lower than fresh tomato fruits, protein and carbohydrate content of the preserved tomato fruits was higher than fresh tomato fruits. Moreover Vitamin C content of the preserved tomato fruits was higher than the fresh tomato fruits.

Trametes polyzona extract increased significantly $(\mathrm{P}>0.05)$ than the fresh fruits. Trametes polyzona extract can therefore be recommended for the preservation of tomato fruits and keeping at refrigerator temperature further enhance the shell life of the fruits.

\section{REFERENCES}

[1]. A.O.A.C. (2012). Official methods of analysis. 19th Edn. Washington DC by Association of official analytical chemist.

[2]. A.P.H.A. (2002). Standard Methods for the Examination of Water and Wastewater. 20th Edition. American Public Health Association. Washington D.C.

[3]. Abdul-Hammed M, Bello, O.S, Azeez M.A. and Adedeji, N. O. (2015.) Bioformation of carotenoids in tomatoes (Solanumlycopersicum) under two ripening conditions: A Kinetic study. Intern. J. Sci. Eng. Res., 6(8): $1-9$.

[4]. Abhinaba G. (2009). Identification of microorganisms responsible for spoilage of tomato (Solanumlycopersicum L.) fruit. J. Phytopath. 1(6):414-416.

[5]. Abugria, D. A. and McElhenney, W. H. (2013). Extraction of total phenolic and flavonoids from edible wild and cultivated medicinal mushrooms as affected by different solvents. Journal of Natural Product and Plant Resources, 3 (3), 37-42.

[6]. Adaskaveg, J. E., Forster, H., and Sommer, N. F. (2002.) Principles of post-harvest pathology and management of decays of edible horticultural crops. In: Post-harvest Technology of Horticultural Crops, 332: 200-250.

[7]. Agbabiaka, T. O., Saliu, B. K., Sule, I.O., Oyeyiola, G.P and Odedina, G.F. (2015). Microbial Deterioration of Tomato Fruit (Lycopersiconesculentum) Sold in Three Popular Markets in Ilorin, Kwara State, Nigeria. Fountain Journal of Natural and Applied Sciences: 4(1): 10-18

[8]. Akinmusire, O.O. (2011). Fungi species associated with the spoilage of some edible fruits in Maiduguri, North eastern Nigeria. Advances in Environmental Biology,.5(1): 157-161. http://www.aensiweb. Com/old/aeb/2011/157-161.

[9]. Akintobi, A. O., Okonko, I.O., Agunbiade, S.O.,Akano, O.R and Onianwa. O (2011). Isolation and identification of fungi associated with the spoilage of some selected fruits in Ibadan South western Nigeria. Academia Arena, Vol.3, No. 11, 110, 2011. http://www.sciencepub.net.

[10]. Alia-Tejacal, 1., Villanueva-Arce, R., PelayoZaldívar, C., Colinas-León, M.T., López-Martínez, V. and Bautista-Banos, S. (2007). Postharvest physiology and technology of sapotemamey fruit (PouteriasapotaOacq.) H.E. Moore \& Steam). Postharvest Biology and Technology, 45: 285-297.

[11]. Alves,M.J., Ferreira I.C.F.R., Dais J., Teixeira V., Martins A andPintado M. (2012). A review on antimicrobial activity of mushroom (Basidiomycetes) extracts and isolated compounds. Plant Med 78:1707-18. 
[12]. Alves M.J., Ferreira I.C.F.R., Froufe H.J.C., Abreu R.M.V., Martins A andPintado M. (2013). Antimicrobial activity of phenolic compounds identified in wild mushrooms, SAR analysis and docking studies. J ApplMicrobiol 115:346-57.

[13]. Ayaz, F.A., HayIrlIoglu-Ayaz S, Alpay-Karaoglu S, Gruz J, Valentová K, Ulrichová J, and Strnad M. (2008.) Phenolic acid contents of kale (Brassica oleraceae L. var. acephala DC.) extracts and their antioxidant and antibacterial activities. Food Chem, 107: 19-25.

[14]. Bai, Y., Lindhout P. (2006). Domestication and breeding of tomatoes: What have we gained and what can we gain in the future? Annals of Botany, 100(5) 1085-1094, doi: 10.1093/aob/mcm 150 .

[15]. Baiyewu, R.A., Amusa, N.A., Ayoola, O.A., and Babalola, O.O. (2007.) Survey of the post harvest diseases and aflatoxin contamination of marketed pawpaw fruit (Carica papaya L) in south western Nigeria. Afr. J. Agric. Sci. 2(4):178-181.

[16]. Balakumar, R., Sivaprakasam, E., Kavitha, D., Sridhar S and Kumar J.S. (2011). Antibacterial and antifungal activity of fruit bodies of Phellinusmushroom extract. Intl J Biosci1:72-7.

[17]. Baldwin, E.A., Scott, J.W., Shewmaker, C.K andSchuch, W. (2000). Flavor trivia and tomato aroma: biochemistry and possible mechanisms for control of important aroma components. HortScience, 35: 1013-1021.

[18]. Barret, D.M and Anthon, G.E. (2008). Color quality of tomato products. American Chemical Society. Chapter 10, 131-139. Acessed at http://ucanr.org/sites/zann_test/files/28712.pdf.

[19]. Barrett, D.M. and Anthon, G. (2001). Lycopene content of California-grown tomato varieties. Acta Horticulture 542: 165-174.

[20]. Bartz, J.A., Sargent, S.A., and Mahovic, M. (2009). Guides to Identifying and Controlling Post Harvest Tomato Disease in Florida. http://www.edis.ifas.ufl.edu/topic_toma_diseases

[21]. Basu, A andImrhan, V. (2006). Tomatoes versus lycopene in oxidative stress and carcinogenesis: conclusions from clinical trials. European Journal of Clinical Nutrition, 1-9.

[22]. Bathgate, B., Goodenough, P.W and Grierson, D. (1986). Regulation of the expression of the psbA gene in tomato fruit chloroplasts and chromoplasts. Journal of Plant Physiology, 24: 223-233.

[23]. Batu, A. (1998). Some factors affecting on determination and measurement of tomato firmness. Turkish Journal of Agriculture and Forestry, 22: 411-418.

[24]. Bello, O.B., Olawuyi, O.J., Azeez, A.H., Adebisi, O.S and Owoade T.A. (2016.) Microorganisms causing post harvest tomato(solanumlycopersicum 1.) fruit decay in nigeria. ScientiaAgriculturae, 13 (2), 93-96. Retrieved fromwww.pscipub.com (DOI: 10.15192/PSCP.SA.2016.13.2.9396).
[25]. Berdegue, J.A., F. Balsevich, L., Flores and T. Reardon, (2005). Central American Supermarket private standards of quality and safety in procurement of fresh fruits and vegetables, Food Policy, 30: 254269.

[26]. Besser, R.E., Lett,S.M.,WeberJ.T and M. Doyle (1993). An outbreak of diarrhea and uremic syndrome from Escherichia coli O157:H7 in fresh pressed apple cider. Journal of the American Medical Association, 269: 2217-2220.

[27]. Beuchat, L.R., (1996). Pathogenic organisms associated with fresh produce. Journal of Food Protection, 59: 204-216.

[28]. Beuchat, L.R., (2002). Ecological factors influencing survival and growth of human pathogens on raw growth of human pathogens on raw fruits and vegetables. Microbiology and Infection, 4: 413-423.

[29]. Beuchat, L.R., (2006). Vectors and conditions for pre-harvest contamination of fruits and vegetables with pathogens capable of causing enteric diseases. Britain food Journal, 108: 38-53.

[30]. Bihn, E.A. and R.B. Gravani, (2006). Role of good Agricultural practices in Fruit and Vegetable safety In: Microbiology of Fresh Produce (Edited by K.R Matthews). Washington DC: ASM Press, pp: 21-28.

[31]. Bok I, M. Madisa, D. Machcha, M. Moamogwe and K. More (2006). Manual for vegetable production in Botswana. Department of Agricultural Research. Gaborone. Botswana

[32]. Bonjar, G.H.S, Nik, A.K and Aghighi S. (2004.) Antibacterial and antifungal survey in plants used in indigenous herbal-medicine of south east regions of Iran. J BiolSci, 4: 405-412.

[33]. Cheesbrough, M., (2006). District Laboratory Practice for Tropical Countries, part 2, second edition. Cambridge University Press.

[34]. Chinedu, S.M and Enya E. (2014.) Isolation of Microorganisms associated with deterioration of tomato(Lycopersiconesculentum) and pawpaw (Carica papaya) fruits. Int. J. Curr. Microbiol. App. Sci. 3(5):501-512.

[35]. Chuku, E.C., Ogbonna, D.N., Onuegbu, B.A. and Adeleke M.T.V. (2008.) Comparative studies on the fungi and biochemical characteristics of snake guard (TrichosanthesCurcumerinalinn) and Tomato (LycopersiconEsculentum Mill) in Rivers State, Nigeria. J. Applied Sci. 8(1): 168-172.

[36]. Chukwuka, K.S., Okonko, I.O andAdekunle, A.A. (2010). Microbial Ecology of organisms causing pawpaw (Carica papaya L.) fruit decay in Oyo State, Nigeria. American-Eurasian Journal of Toxicological Science, Vol.2, No.1, 43-50. http://www/idosi.org/aejts/aejts 2 (1) 10.htm.

[37]. Clewell, D.B. and Dunny, G.M. (2002.) Conjugation and genetic exchange in enterococci. In: Gilmore MS, Clewell DB, Courvalin P, Dunny GM, Murray BE, Rice LB, (Eds.). The enterococci: pathogenesis, molecular biology, and antibiotic resistance. ASM Press, Washington, DC, pp. 265-300 
[38]. Dawczynski, C., Schubert, R., and Jahreis, G. (2007.) Amino acids, fatty acids, and dietary fibre in edible seaweed products. Food Chem. 103:891-899.

[39]. Dorado, J., Van Beek, T. A., Claassen, F. W., and Sierra-Alvarez, R. (2001). Degradation of lipophilic wood extractive constituents in Pinussylvestrisby the white-rot fungi Bjerkanderasp. and Trametesversicolor. Wood Science and Technology, 35 (1-2), 117-125.

[40]. Dorais, M., Ehret, D.L. and Papadopoulos, A. (2008). Tomato (Solanumlycopersicum) health components: fromthe seed to the consumer. Phytochemistry Reviews, 7 (2): 231-250.

[41]. Dudareva N, Pichersky E and Gershenzon J. (2004.) Biochemistry of plant volatilesPlant Physiol, 13518931902. . . : -

[42]. Dundar, A., Acay, H., and Yildiz, A. (2008). Yield performances and nutritional contents of three oyster mushroom species cultivated on wheat stalk. African Journal of Biotechnology, 7 (19), 34973501.

[43]. Effiuwevwere, B.J.O. (2000) Microbial spoilage agents of tropical and assorted fruits and vegetables. Paragraphics publishing company, Port Harcourt, Nigeria. PP 1-39.

[44]. Fagade O.E, Oyelade A.A(2009). A comparative study of the antibacterial activities of some wooddecay fungal to synthetic antibiotic discs. J Environ Agric Food Chem 8:184-8.

[45]. Fajola, A.O. (2006). The Post-Harvest Fruit Rot of Tomato (Lycopersiconesculentum) in Nigeria. Molecular Nutrition and Food Research 23(2): 105109.

[46]. FAO, 2013 World Agriculture Information Centre Data base. Rome,

[47]. Italy.http://www.fao.org/waicent/portal/statistics_en.a sp.

[48]. FAOSTAT (Food and Agricultural organization of the united nations) (2012). Data, various years http://faostat.fao.org/cgi-bin/nph-

db.pl?subset=agriculture. Accessed at 10 April 2012.

[49]. Fatih, K., Tamer, A.U andOskay, M. (2005) Determination of fungi associated with tomatoes (lycopersicumesculentum M.) and tomato pastes. Plant Pathology Journal, 4(4): 146-149, DOI:10.3923/PPi. 2005.146.149.

[50]. Fatima D, Mebrouk K andMiloud H. (2015.)Biopreservation of tomato paste and sauce with Leuconostoc spp. metabolites. Afr. J. Food Sci., 9(6): 359-366

[51]. Fawole, M.O. and Oso, B. A. (2007). Laboratory Manual of Microbiology, Spectrum Books. 1(3): 2535

[52]. Fawole, M.O. and Oso, B.A. (2004). Laboratory Manual of Microbiology. 4th edition. Spectrum Books Limited. pp 1-127.

[53]. Freedman, N.D., Park, Y., Subar, A.F., Hollenbeck, A.R., Leitzmann, M.F., Schatzkin, A., Abnet, C.C. (2008). Fruit and vegetable intake and head and neck Cancer risk in a large United States Prospective Cohort Study. International Journal of Cancer, Vol. 122 No.10, 2330-2336, 2008. DOI:10.1002/ijc.23319.
[54]. Gao Y, Tang W, Gao H, Chan E, Lan J, Li X and Zhou S. (2005). Antimicrobial activity of the medicinal mushroom Ganoderma. Food Rev Intl 21:211-29.

[55]. Geeson, J.D., Browne, K.M., Maddison, K., Shepherd, J. andGuarald, F. (1985). Modified atmosphere packaging to extend the shelf life of tomatoes. Journal of Food Technology, 20: 339-349.

[56]. Gemede, H.F., Haki, G.D., Beyene, F., Woldegiorgis, A.Z., and Rakshit, S. K. (2015). Proximate, mineral, and antinutrient compositions of indigenous Okra (Abelmoschusesculentus) pod accessions: implications for mineral bioavailability. Food Science \& Nutrition 2016; 4(2): 223-233 doi: $10.1002 / f s n 3.282$

[57]. George, B., Kaura, C., Khurdiya, D. S and Kapoor, H. C. (2004.) Anti-oxidants in tomato (Lycopersiumesculentum) as a function of genotype. Indian Food Chemistry, 84: 45-51.

[58]. Gerasimenya V.P., Efremenkova O.V., Kamzolkina O.V., Bogush T.A., Tolstych I.V andZenkova VA. (2002). Antimicrobial and antitoxical action of edible and medicinal mushroom Pleurotusostreatus(Jacq.: Fr.) Kumm extracts. Intl J Med Mush 4:127-32.

[59]. Ghosh A. (2009). Identification of microorganisms responsible for spoilage of tomato (Lycopersicumesculentum) fruit. J. Phytol. 1(6): 414416.

[60]. Giovannucci, E. (1999) Nutritional factors in human cancers. Advances in Experimental Medicine and Biology, 472:29-42.

[61]. Harborne J.B., (2005). Phytochemical methods. A guide to modern techniques of plant analysis (3rd ed.). New Delhi: Springer Pvt Ltd.

[62]. Have, R. T., and Teunissen, J. M. (2001). Oxidative mechanisms involved in lignin degradation by whiterot fungi. Chemical Review, 10, 3397-3413.

[63]. Haydar, A., Mandal,M.A.,Ahmed, M.B Hannan,M.M. Karim,R.,Razvy,M.A., Roy U.K and M. Salahin. (2007). Studies on genetic variability and interrelationship among the different traits in tomato. Middle-East J. Sci. Res., 2(3-4): 139-142.

[64]. Hibbett, D. S. (2006). A phylogenetic overview of the Agaricomycotina. Mycologia, 98 (6), 917-925.

[65]. Ibrahim, A.D., Musa, K., Sani, A., Aliero, A.A and Yusuf B.S. (2011.) Microorganisms associated with the production of volatile compounds in spoilt tomatoes. Res. Biotech. 2(2):82-89.

[66]. Ishikawa N.K., Kasuya M.C.C.M andVanetti M.C.D. (2001). Antibacterial activity of Lentinulaedodesgrown in liquid medium. Brazil J Microbiol 32:206 10.

[67]. Jagadish L.K., Krishnan V.V., Shenbhagaraman R and Kaviyarasan V. (2009). Comparative study on the antioxidant, anticancer and antimicrobial property of Agaricusbisporus(J. E. Lange) Imbach before and after boiling. Afr J Biotechnol 8:654-61.

[68]. James, C., Onarinde, B. A and James, S. J. (2017). The Use and Performance of Household Refrigerators: A Review. Comprehensive Reviews in Food Science and Food Safety 16: 160-179 
[69]. Jay, J.M. (2000). Modern Food Microbiology. 6th edition. Gaithesburg CMD: Aspen. pp 679.

[70]. Joshi, A., Vikram, A and Thakur, M.C(2004). Studies on genetic variability, correlation and path analysis for yield and physico-chemical traits in tomato. Prog. Hort., 36(1): 51-58.

[71]. Kader, A. A. (1992). Post-harvest biology and technology: In: A.A. Kader (ed.) Post-harvest technology of horticultural crops. University of California, Agriculture and Natural Resources, 3311:15-20.

[72]. Kader, A. A. (2002). Post-harvest technology of horticultural crops. University of California, Agriculture and Natural Resources, 3320:535-540.

[73]. Kanetis, L., Förster, H and Adaskaveg, J. E ( 2007.) Comparative efficacy of the new post-harvest fungicides azoxystrobin, fludioxonil, and pyrimethanil for managing citrus green mold. Plant Diseases, 91:1502-1511.

[74]. Knežević, A., Milovanović, I., Stajić, M., andVukojević, J. (2013). Potential of Trametesto degrade lignin. International Biodeterioration\& Biodegradation, 85, 52-56.

[75]. Koroleva O.V., Stepanova, V., Gavrilova V.P., Yakovleva N.S., Landesman, E.O., Yavmetdinov I.S and Yaropolov AI. (2002). Laccase and Mnperoxidase production by Coriolushirsutusstrain 075 in a jar fermentor. J BiosciBioeng,93:449-455.

[76]. Kosanic, M., Rankovic B and Dasic M. (2013.) Antioxidant and antimicrobial properties of mushrooms. Bulg J AgricSci,19: 1040-1046.

[77]. Kumar, R., Mishra, N.K.,Singh, J.,Rai, G.K., Verma, A and M. Rai, (2006). Studies on yield and quality trials in tomato (Solanumlycopersicon (Mill) Wettsd.). Vegetable Sci., 33(2): 126-132.

[78]. Kuzniak, E., Sklodowska, M. (2005) Fungal pathogen-induced changes in the antioxidant systems of leaf peroxisomes from infected tomato plants. Planta, 222:192-200.

[79]. Leonardi, C., Ambrosino, P., Esposito, F. andFogliano, V. (2000). Antioxidative activity and carotenoid and tomatine contents in different typologies of fresh consumption tomatoes. Journal of Agricultural Food Chemistry, 48 (10): 4723-4727.

[80]. Libby, P. (2006). Inflammation and cardiovascular disease mechanisms. The American Journal of Clinical Nutrition, 83: 456S-460S.

[81]. Lindequist, U., Niedermeyer T.H.J and Julich W.D. (2005.) The pharmacological potential of mushrooms-Review. J Evid Based Complementary Altern Med, 2: 285-299.

[82]. Liu, C., Duan, J., Su, Y.C. (2006). Effects of electrolyzed oxidizing water on reducing Listeria monocytogenescontamination on seafood processing surfaces. International Journal of Food Microbiology, 106: 248-253.

[83]. Machuca, A., and Ferraz, A. (2001). Hydrolytic and oxidative enzymes produced by white and brown-rot fungi during Eucalyptus grandisdecay in solid medium. Enzyme and Microbial Technology, 29, 386-391.
[84]. Mahovic, M. J., Steven, A.S and Bartz, J.A (2009). Identifying and Controlling Post Harvest Diseases in Florida http://www.edis.ifas.ufl.edu/topic_tomat _diseases

[85]. Malacrida C, Valle E,and Boggio S. (2006) Postharvest chilling induces oxidative stress response in the dwarf tomato cultivar Micro-Tom. PhysiologiaPlantarum 127:10-18.

[86]. Malundo, T.M.M., Shewfelt, R.L. and Scott, J.W. (1995). Flavor quality of fresh tomato (LycopersicumesculentumMill), as affected by sugar and acid levels. Postharvest Biology and Technology, 6: 103-116.

[87]. Matteo, D., Sacco, A.,Anacleria, M. et al. (2010). The ascorbic acid content of tomato fruits

[88]. is associated with the expression of genes involved in pectin degradation. BMC Plant

[89]. Biology 10(1) : 163.

[90]. Maribe, T. (1995). Advanced Trial of ARP Fresh Market Tomato lines. Africa Regional Program. Unpublished Report pages $81-85$.

[91]. Maul, F., Sargent, S.A., Sims, C.A., Baldwin, E.A., Balaban, M.O. and Huber, D.J. (2000). Tomato flavor and aroma quality as affected by storage temperature. Journal of Food Science, 65: 1228-1237.

[92]. Mohanty, B.K. (2003). Studies on variability, heritability, interrelationship and path analysis in tomato. Annals of Agri. Res., 23(1): 65-69.

[93]. Moretti, C.L., Baldwin, E.A., Sargent, S.A and Huber, D.J. (2002). Internal bruising alters aroma volatile profiles in tomato fruit tissues. HortScience, 37: 378-382.

[94]. Mungai, J., Ouko, J and Heiden, M. (2000). Processing of fruits and vegetables in Kenya. Printed by Agricultural Information Centre. Nairobi. Kenya.

[95]. Murray, P.R., Rosenthal, K.S and Pfaller M.A. (2013.) Medical microbiology, Seventh ed. Elsevier/Saunders, Philadelphia.

[96]. Naika, S., J.vanLidt de Jeude., M, de Goffau., M. Hilmi and B.van Dam. (2005). Cultivation of tomato : production, processing and marketing. CTA. Series no 17

[97]. Neumann, U.P., Berg, T., Baha, M., Puhl, G., Guckelbeger, O., Langreh, J.M and Neuhaus P. (2004.) Long-term outcome of liver transplant for hepatitis C: A 10 year follow-up. Transplant, 77: 226-231

[98]. Nicholson, G. M. (2007). Fighting the global pest problem: Preface to the special toxicant issue on insecticidal toxins and their potential for insect pest control, toxicant, 49:413-422.

[99]. Nikaido H. (2009). Multidrug Resistance in Bacteria. Annu Rev Biochem.78: 119-146.

[100]. Nnamani, C. V., Oselebe,H. O. and Agbautu. A(2009). Assessment of nutritional values of three underutilized indigenous leafy vegetables of Ebonyi state, Nigeria. Afr. J. Biotechnol. 8:2321-2324.

[101]. Nwachukwu E, andUzoeto H.O. (2010). Antimicrobial activity of some local mushrooms on pathogenic isolates. J Med Plant. Res 4:2460-5. 
[102]. Nyirongo, M.K. (1995). Observational Trial of ARP Fresh Market Tomato lines. Africa Regional Program. Unpublished Report pages pp75 - 80

[103]. Ogunbanwo S.T., Fadahunsi, I.F. and Molokwu A.J. (2014.) Thermal stability of lactic acid bacteria metabolites and its application in preservation of tomato pastes. Malays. J. Microbiol., 10(1): 15-23.

[104]. Okezie, B. O. (1998). World food security: the role of post-harvest technology. Food Technology, 52: 6469.

[105]. Oladipo, I.C., Adeleke, D.T and Adebiyi, A.O. (2010). The effect of $\mathrm{pH}$ and Chemical Preservatives on the Growth of Bacteria Isolates from some Nigerian packaged Fruit Juices. Pakistan Journal of Pharmaceutical Science 13:16-21.

[106]. Olutiola, P.O., Famurewa, O and Sonntag H.G., (2000). Introduction to General Microbiology. Practical Approach, $2^{\text {nd }}$ Ediion. Publishing and Media Consults Nigeria.

[107]. Omotosho, O. T. (2005.) Nutritional quality, functional properties and anti-nutrient compositions of the Larva of Cirindaforda (Westwood) (Lepidoptera:saturniidae). J. Zhejiang Univ. Sci. 7:51-55.

[108]. Onuorah, S., Obika, I. and Okafor, U. (2015). Filamentous fungi associated with the spoilage of post-harvest sweet orange fruits (citrus sinensis) sold in Awka Major Markets, Nigeria. Bioengineering and Bioscience,3(3):

44-49 DOI:10.13189/bb.2015.030303

[109]. Oyemaechi, C. U., Chukwuezi, F.O and Ozougwu, V.E.O. (2014). Microbial Agents of Tomato Spoilage in Onitsha Metropolis. Advances in Biological Research 8 (2): 87-93

[110]. Oyetayo, V.O., Okuo, M.O and Olatunji (2010). Inhibitory potentials of ethanolic extract of two wild polyporalesmacrofungi on aerobic microbial isolates of meat. Nigerian Journal of Microbiology, Vol. 24(1): 2033 - 2037.

[111]. Park B.T., Na K.H., Jung N.C., Park J.W and Kim H.H. (2009). Antifungal and anticancer

[112]. activities of a protein from the mushroom Cordycepsmilitaris. Korean J PhysiolPharmacol 13:49-54.

[113]. Prabha, P.L., JannathulFirdous, S.,ThirunethiranKarpagam, G., Banu Karthi1 and Subashini, S.R. (2011). A New Photometric Method of Assay Of Vitamin C In Tomato. International Journal of Institutional Pharmacy and Life Sciences 1(2): 66-74.

[114]. Rahman M and Moon S. (2007). Antimicrobial phenolic derivatives from Dendranthemazawadskiivar. latilobumkitamura(Asteraceae). Arch of Pharm Res, 30:1374-1379.

[115]. Rice, R.P., Rice, L.W and H. D. Tindall. (1990). Fruit and vegetable production in warm climate. The Macmillan Press L.T.D. London and Basingstoke.
[116]. Rosa L.H., Machado KMG., Jacob C.C., Capelari M, Rosa C.A and Zani C.L. (2003). Screening of Brazilian basidiomycetes for antimicrobial activity. Memorias do InstitutoOswaldo Cruz 98:967-74.

[117]. Ruíz, J.J., Alonso, A., García-Martínez, S., Valero, M., Blasco, P. andRuíz-Bevia, F. (2005). Quantitative analysis of flavour volatiles detects differences among closely related traditional cultivars of tomato. Journal of the Science of Food and Agriculture, 85: 54-60.

[118]. Sábio, E., Lozano, M., Montero de Espinosa, V., Mendes, R.L., Pereira, A.P., Palavra, A.F. and Coelho, J.A. (2003). Lycopene and $\beta$-carotene extraction from tomato processing wastes using supercritical CO2. Industrial \& Engineering Chemistry Research, 42 (25): 6641-6646.

[119]. Sahelian, R. (2014). "Saponin in plants benefit and side effects, glycosides and extraction."http://www.raysahelian.com/saponin.html (April.27, 2015).

[120]. Samuel, O. and Orji M.U. (2015). Fungi Associated with the Spoilage of Post-harvest Tomato Fruits Sold in Major Markets in Awka, Nigeria. Universal Journal of Microbiology Research 3(2): 11-16

[121]. Schinor, E.C., Salvador, M.J., Ito, I.Y. and Dias D.A., (2007). Evaluation of the antimicrobial activity of crude extracts and isolated constituents from Chrestascapigera. Brazilian J. MIcrobiol 38: 145-149,

[122]. Seminis-Kenya. (2007). Retrieved from: http://www.freshplaza.com/news

[123]. Shen, H., Shao, S., Chen, J and Zhou, T., (2017). Antimicrobials from Mushrooms for Assuring Food Safety. Comprehensive Reviews in Food Science and Food Safety; 16: 316-329

[124]. Shidfar, F., Froghifar, N., Vafa, M.R., Rajab, A., Hosseini, S., Shidfar, S and Gohari, M. (2010). The effects of tomato consumption on serum glucose, apolipoprotein B, apolipoprotein A-I, homocysteine and blood pressure in type 2 diabetic patients. International Journal of Food Science and Nutrition, 62(3): 289-294, Doi:10.3109/09637486.2010.529072.

[125]. Singh, J.P., Singh D.K and G. Gulshanlal, (2000). Variability pattern in agro-Morphological characters in tomato ( Lycopersiconesculentum L.) Prog. Hort., 32(1):79-81.

[126]. Singleton V.L., Orthofor R andLamuela-Raventos R.M. (1999). Analysis of total phenols and other oxidation substrates and antioxidants by means of Folin-Cioalteau Reagents. Methods Enzymol. 299:152-178

[127]. Skrede, I., Engh, I. B., Binder, M., Carlsen, T., Kauserud, H andBendiksby, M. (2011). Evolutionary history of Serpulaceae(Basidiomycota): Molecular phylogeny, historical biogeography and evidence for a single transition of nutrition mode. Evolutionary Biology, 11 (1). doi:10.1186/1471-2148-11-230

[128]. Slimestad, R andVerheul, M.J. (2005). Content of chalconaringenin and chlorogenic acid in cherry tomatoes is strongly reduced during postharvest ripening. Journal of Agricultural and Food Chemistry, 53 (18): 7251-7256. 
[129]. Soto, M. L., Moure, A., Dominguez, H., andParajó, J. C. (2011). Recovery, concentration and purification of phenolic compounds by adsorption: A review. Journal of Food Engineering, 105, 1-27.

[130]. Sridhar S, Sivaprakasam E, Balakumar RandKavitha D. (2011). Evaluation of antibacterial and antifungal of Ganodermalucidum(Curtis) P. Karst fruit bodies extracts. West J SciTechnol1:8-11.

[131]. Stevens, M.A. (1974). Varietal influence on nutritional value. P. 87 - 110. In P.L. White and N.SELVEY (eds.) Nutritional qualities of fresh fruits and vegetables. Futura Publ. Co., Mt. Kisco, N.Y.

[132]. Sun, F.-.H., Li, J., Yuan, Y.-X., Yan, Z.-Y and Liu, X.-F. (2011). Effect of biological pretreatment with Trameteshirsutayj9 on enzymatic hydrolysis of corn stover. International Biodeterioration and Biodegradation, 65 (7), 931-938.

[133]. Taleb-Contini S.H., Salvador, M.J., Watanabe, E. Ito, I.Y. and Oliveira DCR. (2003.) Antimicrobial activity of flavonoids and steroids isolated from two Chromolaenaspecies. Braz J Pharm Sci,39: 403-408

[134]. Talvas, J., Caris-veyrat, C., Guy, L., Rambeau, M., Lyan, B., Minet-Quinard, R., Lobaccaro, J.A, Vasson, M., George, S., Mazur,A and Rock, E., (2010). Differential effects of lycopene consumed in tomato paste and lycopene in the form of a purified extract on target genes of cancer prostatic cells. American Journal of Clinical Nutrition, 91(6): 1716-1724 doi: 10.8945/ajcn.2009.28666.

[135]. Thatoi H.N and Singdevsachan S.K. (2014). Diversity, nutritional composition and medicinal potential of Indian mushrooms: A review. Afr $J$ Biotechnol,13: 523-545.

[136]. Thirupathi, V., Sasikala, S and John Kennedy, Z. (2006). Preservation of fruits and vegetables by wax coating. Journal of science and food agriculture, 55:1-10.

[137]. Tibuhwa, D. D. (2012). Antiradical and antioxidant activities of methanolic extracts of indigenous termitarian mushroom from Tanzania. Food Science and Quality Management, 7, 13-23.

[138]. Upadhyay, M.J.P., Singh, A and Joshi, A(2005). Studies on genetic variability in tomato (Lycopersiconesculentum Mill.). Prog. Hort. 37(2): $463-465$

[139]. Valentin, L., Kluczek-Turpeinen, B., Oivanen, P., Hatakka, A., Steffen, K., andTuomela, M. (2009). Evaluation of basidiomycetous fungi for pretreatment of contaminated soil. Journal of Chemical Technology and Biotechnology, 84, 851-858.

[140]. Van der Vooren, J., G.W.H. Welles, and G. Hay man. (1986.) Glasshouse crop production, pp 581623. In: J.G. Atherton and J. Rudich (eds.). The tomato crop. Chapman and Hall, London.

[141]. Varela, A.M.,Seif, A andLohr. B(2003). A guide to IPM in tomato production in Eastern and Southern Africa. CTA/ICIPE/GTZ.

[142]. Veershetty, V. (2004). Studies on variability, character association and genetic diversity in tomato (Lycopersiconesculentum Mill). M.Sc. (Agric.) Thesis, Uni. Agric. Sci., Dharwad (India).
[143]. Vu, T.S., Smout, C., Sila, D.N., LyNguyen, B., Van Loey, A.M.L. andHendrickx, M.E.G. (2004). Effect of preheating on thermal degradation kinetics of carrot texture. Innovative Food Science and Emerging Technologies, 5: 37-44.

[144]. Wamache, A. (2005.) Vegetable seeds handbook. Regina seeds Seminis. Printed by Bizone ltd. Nairobi Kenya, 23-25.

[145]. Wogu MD, and Ofuase O. (2014.) Microorganisms responsible for the spoilage of tomato fruits, Lycopersicumesculentum, sold in markets in Benin City, Southern Nigeria. Sch. Acad. J. Biosci. 2(7):459-466.

[146]. Yan, Z., Kang, T., Sicong, T., Ming, L., Jing, S., Yuepeng, H. (2010). A combination of heat treatment and Pichiaguilliermondii prevents cherry Tomato Spoilage by Fungi. International Journal of Food Microbiology 137(1):106-110.

[147]. Yeung, D.L. andLaquatra, I. (2003). Heinz Handbook of Nutrition, 9th Edition - Heinz Corporate Research Center.

[148]. Zdenka, P., Peter, S., Hana, D and Milan, H. (2010). Antimutagenic effects of Lycopene and Tomato Puree. Journal of Medicine and Food, Vol.13, No.6, 1443-1450, DOI: 10.1089/jmf.2009.0277.

[149]. Zhang, C., Suzanne, C., Ho, Y., Chen, J., Fu, S., Cheng, F and Lin.(2009). Greater vegetable and fruit intake is associated with a lower risk of breast cancer among Chinese Women. International Journal of Cancer, 125(1): 181-188, DOI:10.1002/ijc.24358.

[150]. Ziarati, $P$ andGhasemynezhad-Shanderman, S.-.S. (2014). Mineral content in Pleurotus(Oyster mushroom): Association of cooking method. International Journal of Plant, Animal and Environmental Sciences, 4 (2), 496-501.

[151]. Zjawiony J.K. (2004). Biologically active compounds from Aphyllophorales (Polypore) fungi. J Nat Prod 67:300-10. 